\title{
Pharmacokinetics, Safety, Efficacy, and Biomarker Profiles During Nemolizumab Treatment of Atopic Dermatitis in Adolescents
}

\author{
Robert Sidbury · Sady Alpizar · Vivian Laquer · Sunil Dhawan • \\ William Abramovits · Luca Loprete · Jayendar Kumar Krishnaswamy • \\ Faiz Ahmad $\cdot$ Zarif Jabbar-Lopez $\cdot$ Christophe Piketty (D)
}

Received: November 1, 2021 / Accepted: December 27, 2021 / Published online: January 28, 2022

(c) The Author(s) 2022

\begin{abstract}
Introduction: Nemolizumab, a new monoclonal antibody that targets the receptor alpha of the neuroimmune cytokine interleukin-31 (IL-31), has shown efficacy in atopic dermatitis (AD) in adults. This study evaluated the pharmacokinetics (PK) and safety of nemolizumab in adolescents with moderate to severe $\mathrm{AD}$ as well as the relationship between nemolizumab concentrations and clinical efficacy and the effect of nemolizumab on protein biomarkers. Methods: Open-label, 16-week study of nemolizumab in patients aged 12-17 years with moderate to severe $\mathrm{AD}$ (baseline $\mathrm{EASI} \geq 16$, IGA $\geq 3$, and BSA $\geq 10 \%$ ) and associated pruritus with baseline average daily peak pruritus
\end{abstract}

Supplementary Information The online version contains supplementary material available at https:// doi.org/10.1007/s13555-021-00678-7.

R. Sidbury $(\square)$

Departments of Pediatrics and Dermatology, Seattle Children's Hospital, 4800 Sand Point Way NE

OC.9.835, Seattle, WA 98105, USA

e-mail: Robert.sidbury@seattlechildrens.org

S. Alpizar

Clinical Research Trials of Florida, Tampa, FL, USA

V. Laquer

First OC Dermatology, Fountain Valley, CA, USA numeric rating scale (PP-NRS) intensity of at least 4. Nemolizumab was administered subcutaneously as a loading dose of $60 \mathrm{mg}$ at baseline, followed by $30 \mathrm{mg}$ every 4 weeks until week 12 with background topical corticosteroids (TCS) or calcineurin inhibitors (TCI). Subsequently patients were followed for 8 weeks more. Stratum corneum (SC) and plasma samples were collected for biomarker assessments.

Results: Twenty patients participated, with a mean age of $14.8 \pm 1.6$ years. The PK of nemolizumab was described by a one-compartment model with linear elimination, a first-order absorption, and a mean half-life of $16.7 \pm 4.1$ days. Mean trough concentrations ranged from $2935 \pm 1029 \mathrm{ng} / \mathrm{mL}$ to $3292 \pm 2018 \mathrm{ng} / \mathrm{mL}$ over the 16-week treatment period. There was a marked improvement in rash, itch, and sleep with a decrease from baseline to week 16 in EASI by $66.5 \pm 32.5 \%$, in PP-NRS by $43.2 \pm 37 \%$, and in sleep disturbance

\author{
S. Dhawan \\ Center for Dermatology Clinical Research, Fremont, \\ CA, USA \\ W. Abramovits \\ Dermatology Treatment and Research Center, \\ Dallas, TX, USA \\ L. Loprete · J. K. Krishnaswamy · Z. Jabbar-Lopez . \\ C. Piketty \\ Galderma R\&D, Entre-deux-villes, Switzerland \\ F. Ahmad \\ Galderma Laboratories, LP, Fort Worth, TX, USA
}


numeric rating scale by $53.5 \pm 47.8 \%$. The popPK and PK/PD analyses confirmed that model-predicted exposure and efficacy of nemolizumab were similar in adolescents compared to adults receiving the same dosing regimens. Age did not impact PK parameters, while the main source of PK variability was body weight. Analyses of SC samples identified a panel of AD-related pro-inflammatory biomarkers that were upregulated in lesional skin (compared to non-lesional skin) and correspondingly downregulated in clinical responders to nemolizumab (based on EASI75 and PPNRS $\geq 4$ ). Four biomarkers (CCL20, CCL22, CCL27, and VEGF) had changes that were 1.9-3.5-fold higher in EASI responders than in EASI non-responders (all $p<0.05$ ). Analysis showed no significant correlation between plasma biomarkers and clinical scores. Adverse events were experienced by $33.3 \%$ of subjects $(n=6)$ and were primarily mild or moderate in severity.

Conclusions: Nemolizumab PK and safety profiles in adolescents with moderate to severe $\mathrm{AD}$ are consistent with previous nemolizumab studies in adults. PK/PD models demonstrate similar exposure-response profiles in 12- to 17-year-old adolescents and adults for three clinical endpoints (EASI, IGA, and PP-NRS). Nemolizumab treatment reversed AD-related pro-inflammatory biomarkers in skin, indicating that the neuroimmune cytokine IL-31 is an important mediator of multiple pathways in AD.

Clinical Trial Registration: Clinicaltrials.gov NCT03921411.

Keywords: Nemolizumab; Atopic dermatitis; Biomarkers; Pharmacokinetics; Adolescents

\section{Key Summary Points}

Nemolizumab, a monoclonal antibody that targets the receptor alpha of the neuroimmune cytokine IL-31, has shown efficacy in atopic dermatitis (AD) in adults.
This study evaluated the pharmacokinetics (PK) and safety of nemolizumab in adolescents with $\mathrm{AD}$ and the effect of nemolizumab on protein biomarkers.

Nemolizumab PK and safety profiles in adolescents with moderate to severe AD are consistent with previous nemolizumab studies in adults and nemolizumab treatment reversed AD-related proinflammatory biomarkers in skin, indicating that the neuroimmune cytokine IL-31 is an important mediator of multiple pathways in AD.

\section{INTRODUCTION}

Atopic dermatitis (AD) is a common inflammatory skin disease, affecting $10-20 \%$ of the population and as many as $25 \%$ of children [1-3]. Chronic pruritus can be considered a diseasedefining symptom of $\mathrm{AD}$; additional characteristic signs and symptoms include xerosis, and eczematous lesions that may manifest as erythema, infiltration/papulation, oozing/crusting, excoriations, and lichenification [3, 4]. Notably, approximately $30 \%$ of children and $50 \%$ of adult patients with AD have moderate to severe disease [5-7]. Topical treatment with corticosteroids or calcineurin inhibitors can be efficacious, especially in milder $\mathrm{AD}$ [4]; proactive therapy with topical corticosteroid (TCS) and tacrolimus is recommended by recent guidelines for therapy of $\mathrm{AD}$ [8]. However, topical therapy may not always be sufficient to control moderate to severe AD [9]. Systemic immunosuppressive treatments such as cyclosporine, methotrexate, mycophenylate or azathioprine are more efficacious, but are often accompanied by adverse effects [4]. In recent years, knowledge of the pathophysiologic factors in $\mathrm{AD}$ has increased, and a variety of new treatments have either received regulatory approval or are undergoing clinical evaluation for efficacy and safety, including nemolizumab [4]. The first 
drug approved by the US Food and Drug Administration (FDA) for treatment of moderate to severe $\mathrm{AD}$ was dupilumab in December 2020. Dupilumab is approved for adolescents with moderate to severe AD eligible for systemic therapy and can currently be considered as a first-choice systemic treatment in this population $[10,11]$.

Nemolizumab, a new monoclonal antibody that targets the receptor alpha of the neuroimmune cytokine interleukin-31 (IL-31), has shown efficacy in AD in adults [12-14]. In phase 2 studies of adults with moderate to severe $\mathrm{AD}$, nemolizumab improved $\mathrm{AD}$ lesions and pruritus with a favorable safety profile [15-17]. $\mathrm{AD}$ is most common in childhood but there are limited data with nemolizumab in patients aged younger than 18 . The primary objective of this study was to evaluate the pharmacokinetics (PK) and safety of nemolizumab in adolescents with moderate to severe AD. Secondary objectives were to evaluate the relationship between nemolizumab systemic exposure and clinical efficacy endpoints (pharmacokinetic/pharmacodynamic relationship) and the effect of nemolizumab therapy on protein biomarkers as an exploratory endpoint.

\section{METHODS}

This was an open-label, 16-week study of nemolizumab in patients aged $12-17$ years with moderate to severe $\mathrm{AD}$ and associated pruritus not adequately controlled with topical therapy. Nemolizumab was administered subcutaneously as a loading dose of $60 \mathrm{mg}$ at baseline, followed by $30 \mathrm{mg}$ every 4 weeks until week 12 with background TCS treatment; the treatment period ended at week 16 and patients were followed for an additional 8 weeks. Additional details on the study methodology are provided in the Supplementary Material.

\section{Ethics Approval}

Ethics committee approval was provided by ADVARRA, 6940 Columbia Gateway Drive Suite\#110, Columbia, MD 21046 USA. This study was conducted in accordance with the accepted version of the Declaration of Helsinki and all relevant federal regulations as set forth in Parts 50, 56, 312, Subpart D, of Title 21 of the US Code of Federal Regulations, in compliance with International Council for Harmonization (ICH) Good Clinical Practice (GCP) guidelines, and according to the appropriate regulatory requirements in the countries where the study was conducted. Before any study procedures were performed, each subject (or their legally acceptable representative[s] if applicable) voluntarily signed and dated the informed consent form (ICF, or assent form, as applicable).

\section{Study Participants}

In addition to inadequate response to topical $\mathrm{AD}$ medications, the following disease-severity criteria were required for eligibility: baseline Eczema Area and Severity Index (EASI) score $\geq 16$, Investigator Global Assessment (IGA) score of 3 or higher, and AD body surface area (BSA) involvement of at least $10 \%$; AD-associated pruritus was indicated by a baseline average daily peak pruritus numeric rating scale (PPNRS) intensity of at least 4 . The primary exclusion criteria were body weight $<30 \mathrm{~kg}$, poorly controlled asthma or current history of chronic obstructive pulmonary disease and/or chronic bronchitis, and history of failure on another biologic or JAK inhibitor.

\section{Rescue Therapy}

Rescue therapy could be prescribed to subjects at any time during the study (except the run-in period) as deemed necessary by the investigator. As a general guideline, rescue therapy was not prescribed within the first 2 weeks after baseline to allow assessment of study drug exposure. Rescue therapy was directed at AD, and included topical calcineurin inhibitors, higher potency TCS, oral corticosteroids, biologics, phototherapy, or systemic nonsteroidal immunosuppressants/immunomodulators. If rescue therapy was topical or phototherapy, study drug administration continued; study drug was permanently discontinued if systemic rescue was required. 


\section{Efficacy and Safety Assessments}

The primary objective of the study was to evaluate the safety and pharmacokinetics of nemolizumab; however, efficacy assessments were performed including EASI scoring, IGA scores $(0=$ none to $4=$ severe, with treatment success defined as a score of $0 / 1$ or clear/almost clear), BSA, and the SCORing Atopic Dermatitis (SCORAD) measure. Changes in itch and sleep were also captured, using the PP-NRS and a sleep disturbance NRS (SD-NRS), both scales from $0=$ none to $10=$ worst imaginable. Safety evaluations were conducted at every visit and included collection of adverse events, physical examination, and laboratory assessments.

\section{Pharmacokinetic Assessments}

Blood samples to determine the PK profile of nemolizumab were collected. Serum concentration was assessed at baseline, weeks 1-2, 4, 8, 12,16 , and 24 , and determined using a validated enzyme-linked immunosorbent assay (ELISA) method with a quantification limit of 100 ng/mL (Shin Nippon Biomedical Laboratories, Japan).

A first-order absorption and one-compartment distribution population PK (popPK) model was initially developed using nemolizumab serum concentrations from three clinical studies conducted in 407 adults. The studies assessed nemolizumab administered subcutaneously over the dose range of $0.1-3 \mathrm{mg} / \mathrm{kg}$ and $10-90 \mathrm{mg}$ for weight-based and flat dosing, respectively. The effect of several covariates including age, body weight, serum creatinine, estimated glomerular filtration rate, bilirubin, serum albumin, total protein, IgE, sex, and clinical study was explored. The final model identified in the adult population was used to derive individual empirical Bayes estimates of model-derived PK parameters in the adolescent population, including maximum/trough concentrations $\left(C_{\max }, C_{\text {trough }}\right)$, area under the curve (AUC), volume of distribution $\left(V_{\mathrm{d}} / F\right)$, oral clearance $(C L / F)$, and absorption rate $\left(k_{\mathrm{a}}\right)$.

Three pharmacokinetic/pharmacodynamic (PK/PD) models were developed to correlate the concentration of nemolizumab to clinical efficacy. The PK/PD models were applied to the following PD endpoints: EASI, IGA, and weekly average PP-NRS.

\section{Immunogenicity Assessment}

Serum samples to assess anti-drug antibodies (ADA) were assessed at baseline, weeks 4, 8, 12, 16 , and 24 , and determined using a validated electrochemiluminescence immunoassay (ECLIA) method (LSI Medience Corporation, Japan).

\section{Biomarker Assessments}

The effect of nemolizumab was investigated on 30 selected biomarkers relevant to $\mathrm{AD}$ on both plasma and stratum corneum (SC) samples [15]. Plasma samples were collected at baseline and weeks 8 and 16. Samples of the SC were collected at baseline and week 16 via tape strips (DSquame). Two lesional and two non-lesional areas were identified at baseline and four tape strips were collected from each area (16 total). At week 16, samples were collected only from the two lesional areas identified at baseline (eight strips in total). Levels of proteins were associated with clinical parameters (75\% response on EASI [EASI75], IGA $\leq 1$, PP-NRS decrease by at least 4 points).

\section{Statistical Methods}

All efficacy analyses used the intent-to-treat (ITT) population which included all enrolled subjects who signed a consent form; all safety data were summarized using the safety population which included all subjects who were administered at least one dose of study medication; all pharmacokinetic analyses were performed using the PK population including all subjects who provided at least one post-baseline evaluable drug concentration value. Subject disposition, demographics, baseline characteristics, previous therapies, and concomitant therapies were summarized by descriptive statistics. Efficacy variables were summarized by analysis visit from baseline unless specified 
otherwise. Efficacy analyses were performed using last observation carried forward (LOCF) or non-responder imputation. Efficacy data after use of rescue medication were treated as treatment failure and were set to missing for analysis. Adverse events were summarized using the number and percentage of subjects reporting each system organ class and preferred term. Changes in physical examination were summarized by body system and visit from baseline. Changes in laboratory assessments were summarized by descriptive statistics by visit.

Nemolizumab serum concentration data (in nanograms per milliliter) were summarized by visit from baseline to week 24 using the following statistics: $n$, arithmetic mean, standard deviation, $\% \mathrm{CV}$, geometric mean, \%CV geometric mean, median, minimum, maximum, 95\% CI of mean and number of BLQs (below limit of quantification). Estimates of popPK parameters $\left(C l / F, V_{\mathrm{d}} / F, k_{\mathrm{a}}\right)$, including inter-individual variability, IIV), covariate effects, residual error and their relative standard error (RSE) were presented. Individual derived PK parameters at steady state were summarized using the following statistics: $n$, arithmetic mean, SD, geometric mean, \%CV, median, minimum, maximum and 95\% CI. All pharmacokinetic analyses were based on the observed cases, and no missing data were imputed.

Primary inference for all biomarker analyses was based on the observed cases. All biomarker variables (e.g., absolute and change from baseline) were summarized descriptively at each time point. In addition, a box plot was produced at each time point. If the variable did not have a Gaussian distribution, it was $\log$ transformed.

\section{RESULTS}

\section{Subject Disposition and Demographics}

A total of 20 subjects were enrolled (ITT population), of which 18 subjects were treated at least once and were included in the safety and PK populations. Two subjects who did not meet inclusion criteria and were enrolled in error received no doses of nemolizumab. Thirteen
Table 1 Demographic information and baseline disease characteristics

\begin{tabular}{ll}
\hline & $\begin{array}{l}\text { Nemolizumab 30 mg (ITT } \\
\text { population, } N=\mathbf{2 0})\end{array}$ \\
\hline Gender $(n, \%)$ & $8(40 \%)$ \\
Male & $12(60 \%)$ \\
Female & \\
Race & $2(10 \%)$ \\
Asian & $10(50 \%)$ \\
Black/African American & $7(35 \%)$ \\
Caucasian & $1(5 \%)$ \\
Other & \\
Weight & $69.1 \pm 17.5$ \\
Mean \pm SD & \\
Disease characteristics & $25.2 \pm 7.2$ \\
EASI score (mean \pm SD) & $15,75 \%$ \\
IGA 3 ( $n$, \%) & $5,25 \%$ \\
IGA 4 $(n, \%)$ & $41.3 \%$ \\
Mean BSA affected $(\%)$ & $6.9 \pm 1.7$ \\
PP-NRS (mean \pm SD) & $5.6 \pm 2.3$ \\
SD-NRS (mean \pm SD) & $63.0 \pm 11.3$ \\
SCORAD (mean $\pm S D)$ & \\
\hline
\end{tabular}

$S D$ standard deviation

subjects received all treatment doses (completers), and five subjects discontinued early (three because of adverse events and two because of protocol deviations).

Demographic and disease characteristic information are presented in Table 1 . Subjects in the ITT population had a mean age of $14.8 \pm 1.6$ years and body weights ranging from 49 to $112 \mathrm{~kg}$. Ten subjects $(50.0 \%)$ had a medical history of stable asthma at baseline.

\section{Pharmacokinetics}

Peak nemolizumab serum concentrations were observed either at week 1 or week 2 visits after 
the 60-mg loading dose. Maximum observed concentrations ranged from 2550 to $11,100 \mathrm{ng} /$ $\mathrm{mL}$, with a mean value of $6533 \pm 2330 \mathrm{ng} / \mathrm{mL}$. Steady state concentrations were achieved by week 4, with mean observed trough concentrations ranging from $2935 \pm 1029 \mathrm{ng} / \mathrm{mL}$ to $3292 \pm 2018 \mathrm{ng} / \mathrm{mL}$ over the 16 -week treatment period. At the end of the treatment period (week 16), nemolizumab was eliminated from the serum with an average half-life of $16.7 \pm 4.1$ days. As shown in Fig. 1 , average nemolizumab observed exposures in adolescents were comparable to those in adults receiving the same dosing regimens.

A population pharmacokinetic (popPK) model developed from adult data was used to describe the time course of nemolizumab exposure and investigate sources of variability in the PK profile of nemolizumab (details of the model are provided in the Supplementary Material). The pharmacokinetics of nemolizumab were described by a one-compartment model with linear elimination, a first-order absorption, and a lag time. A moderate impact of the dose was identified on the bioavailability with an increase of bioavailability with decreasing dose. Body weight was confirmed as the main source of PK variability, impacting both elimination $(\mathrm{CL} / F)$ and distribution $(V / F)$, with increasing body weight translating to decreased systemic exposure. The albumin plasma concentrations moderately impacted
$\mathrm{CL} / F$. Estimates of the main population $\mathrm{PK}$ parameters in adults are reported in Table 2.

The adult model was then used to predict the nemolizumab individual PK profile in

Table 2 Nemolizumab serum pharmacokinetic parameters estimated with popPK analysis (adolescent PK population, $N=18$ )

\begin{tabular}{clc}
\hline Parameter & $\begin{array}{l}\text { Population } \\
\text { estimate in } \\
\text { adults }\end{array}$ & $\begin{array}{l}\text { Mean }( \pm \text { SD) of } \\
\text { individual estimates in } \\
\text { adolescents }\end{array}$ \\
\hline $\mathrm{CL} /$ & 0.324 & $0.325(0.153)$ \\
$F(\mathrm{~L} /$ day $)$ & & $7.15(2.24)$ \\
$V_{\mathrm{d}} / F(\mathrm{~L})$ & 7.46 & $16.7(4.11)$ \\
$t_{1 / 2}($ day $)$ & 16.0 & $0.402(0.145)$ \\
$k_{\mathrm{a}}(1 /$ day $)$ & 0.592 & $5975.5(2175.2)$ \\
$\begin{array}{c}C_{\mathrm{max}}(\mathrm{ng} / \\
\mathrm{mL}\end{array}$ & $\mathrm{NC}$ & $111.4(48.0)$ \\
$\mathrm{AUC}_{\text {inf }}$ & $\mathrm{NC}$ & \\
$\begin{array}{c}\left(\mu \mathrm{g}^{*} \text { day/ }\right. \\
\mathrm{mL}\end{array}$ & & \\
\hline
\end{tabular}

$N C$ not calculated, $C L / F$ apparent clearance after extravascular administration, $V_{d} / F$ apparent volume of distribution after extravascular administration, $k_{a}$ firstorder constant of absorption, $t_{1 / 2}$ terminal half-life, $C_{\max }$ maximum drug concentration, $A U C_{\text {inf }}$ area under the concentration-time curve to infinity

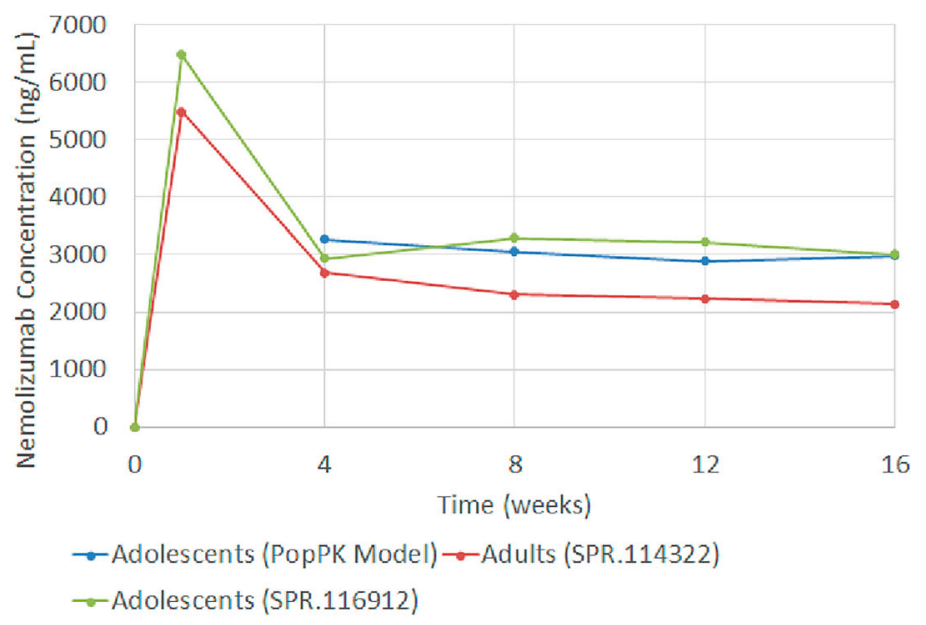

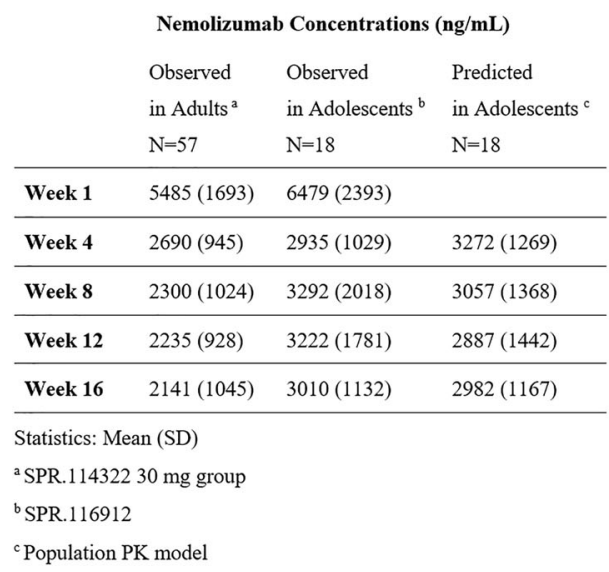

Fig. 1 Average predicted and observed nemolizumab concentrations in adolescents and adults 
adolescents using their baseline characteristics (age, body weight, albumin levels) and dosing history and then compared to the observed data. As shown in Table 2 and Fig. 1, the popPK model developed from adult data was able to accurately predict nemolizumab PK in adolescents. No impact of age was observed on estimates for systemic clearance, volume of distribution, constant of absorption, or terminal half-life obtained with popPK modeling.

\section{Immunogenicity}

Overall incidence of ADA formation in the adolescent population was limited, as only 1 of 18 subjects presented treatment-related ADA at week 4. No ADA-positive sample had neutralizing antibodies. Importantly, serum nemolizumab concentrations in ADA-positive subjects were not different from those in ADA-negative subjects.

\section{Efficacy}

There was an improvement in $\mathrm{AD}$ signs and symptoms in subjects treated with nemolizumab. The mean EASI score was reduced from $25.2 \pm 7.2$ at baseline to $8.0 \pm 9.0$ at week 16 , a $66.5 \%$ (32.5) improvement (Fig. 2). In addition, $9 / 15(60 \%)$ of subjects had a $75 \%$ response on EASI (EASI75) score at week 16. Improvement in the mean IGA was observed beginning at week 4 and continued to increase through week 16 . By week 16, seven subjects (35\%) achieved IGA success $(0 / 1$ or clear/almost clear plus $\geq 2$ grade improvement). The mean SCORAD scores were reduced from 63.0 at baseline to 30.0 at week 16, a 50\% reduction. Concomitantly, there was a $-53 \%$ reduction in mean BSA involvement from baseline to week 16, from $41.3 \%$ at baseline to $19.4 \%$ at week 16 .

Improvements in pruritus were indicated by PP-NRS and SD-NRS. There was an improvement in PP-NRS by $43.2 \pm 37 \%$ (from 6.9 at baseline to 3.8 at week 16). By week 16, 5/10 $(50 \%)$ of subjects were judged PP-NRS responders, defined as a weekly improvement in PPNRS score $\geq 4$ points. Better quality sleep was demonstrated by a mean $-53.5 \%$ change from baseline in SD-NRS.

PK/PD analyses showed that model-predicted efficacy (described by IGA, EASI, and PPNRS clinical assessments) was similar in adolescents compared to adults (details of the modeling are provided in the Supplementary Material).

\section{Biomarker Analyses}

Supervised and unsupervised analyses (see Supplemental Methods) on 30 AD-relevant biomarkers were performed. In the supervised analysis, six proteins were identified that were significantly associated with EASI75 response: CCL18, CCL20, CCL22, CCL27, IL1RA with false discovery rate $(\mathrm{FDR})<0.05$, and VEGF-A with FDR $<0.10$, where the FDR considered is the minimum adjusted $p$ value (interaction, main effect). Four of these biomarkers (CCL20, CCL22, CCL27, and VEGF) had changes that were 1.9-3.5-fold higher in EASI responders

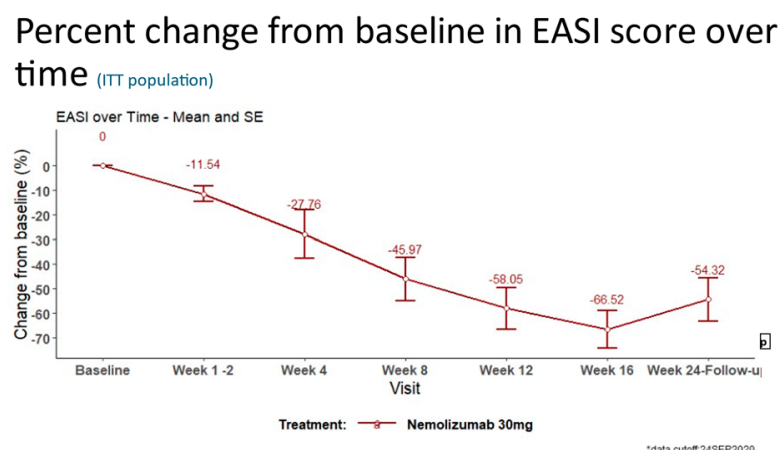

Fig. 2 Percentage change from baseline in EASI score at each study visit. ITT population 
than in EASI non-responders (all $p<0.05$, Fig. 3). Although not significant from an FDR criterion, CCL17 was also significantly different between lesional and non-lesional skin in EASI75 responders and was concomitantly reduced post-treatment in week 16 lesional skin. Further, CCL17 and CCL20 expression in the SC were significantly associated with PPNRS (Fig. 4). Finally, unsupervised analysis identified 15 proteins (including the six proteins found by the supervised analyses) that were significantly associated with EASI75 $(p=0.0041 ;$ Fig. 5). These included CCL18,
CCL20, CCL22, CCL27, CX3CL1, CXCL6, CXCL8, CYSTATIN, FASL, GALECTIN, IL-11, IL21, IL-1RA, SPD, VEGF-A (Fig. 5). Biomarker analysis showed no significant correlation between the measured proteins in plasma and clinical scores (data not shown).

\section{Safety}

Adverse events were experienced by $33.3 \%$ of subjects $(n=6)$ and were primarily mild or moderate in severity. There were no deaths and three serious adverse events occurred in two
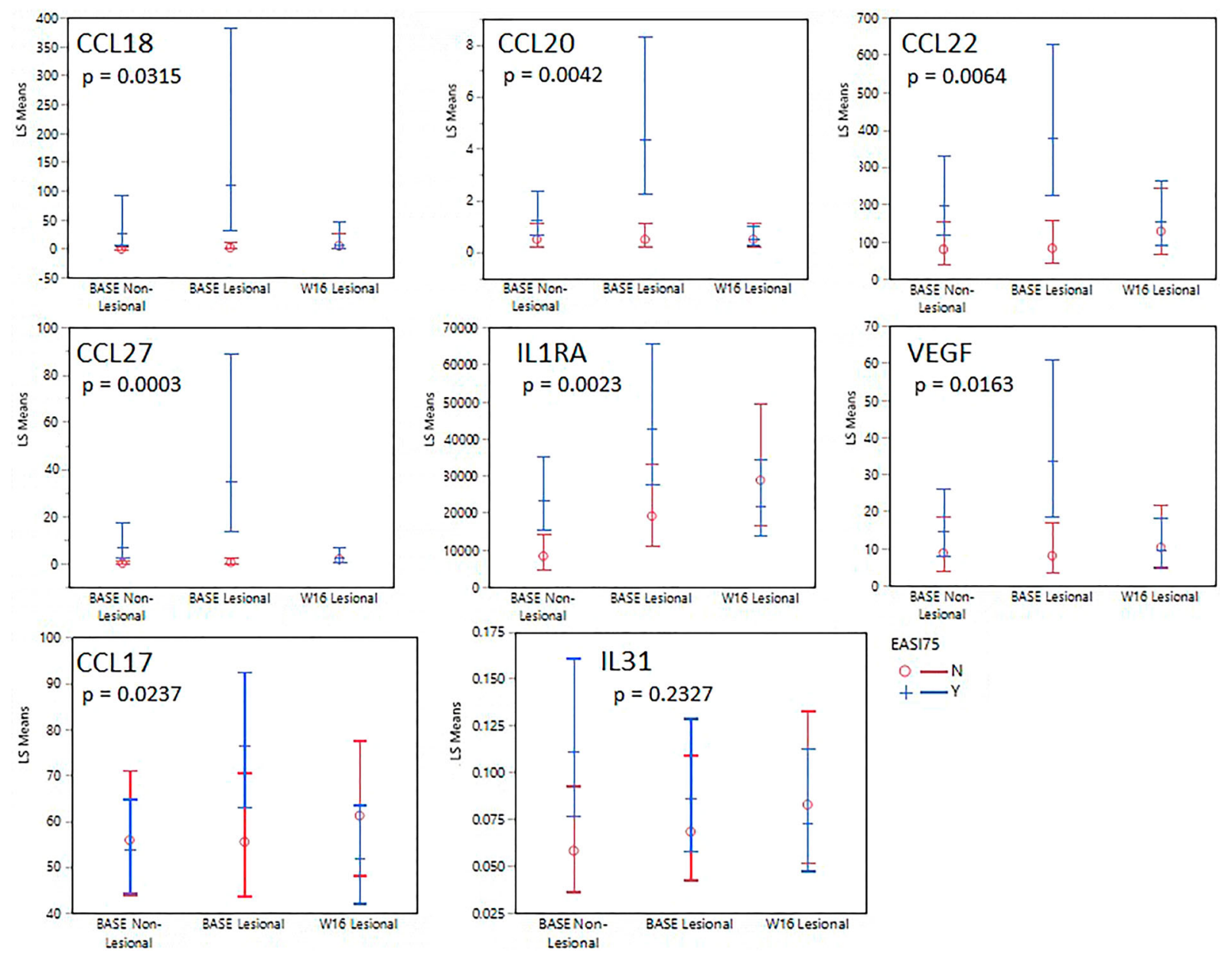

Fig. 3 Supervised analysis by mixed model repeated measures (MMRM) of significantly regulated protein biomarkers in stratum corneum with respect to EASI75. The $p$ value corresponds to the interaction between sample type and EASI75 responders (Y; blue line) vs non responders $(\mathrm{N}$; red line). Least-square estimated means
(LS means) are shown with 95\% confidence intervals for each treatment in each skin condition (lesional/non lesional baseline and week 16). For simplicity, only the interaction $p$ value is mentioned in the figure, but the selection criterion is based on the minimum adjusted $p$ value (interaction, main effect) 


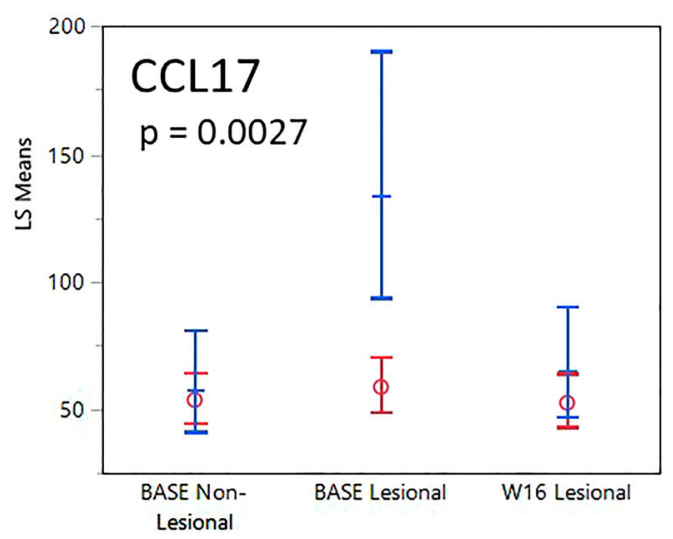

Fig. 4 Supervised analysis (MMRM) of the two significantly regulated protein biomarkers CCL17 and CCL20, in stratum corneum with respect to PP-NRS at week 12. The $p$ value corresponds to the interaction between sample type and PP-NRS responders (Y; blue line) vs non responders $(\mathrm{N}$; red line). Least-square estimated means (LS

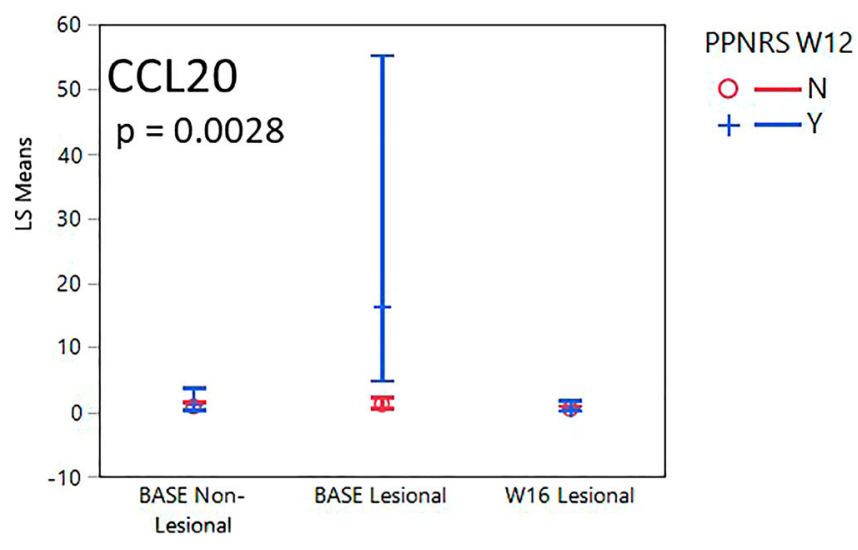

means) are shown with 95\% confidence intervals for each treatment in each skin condition (lesional/non-lesional baseline and week 16). For simplicity, only the interaction $p$ value is mentioned in the figure, but the selection criterion is based on the minimum adjusted $p$ value (interaction, main effect)

\section{Unsupervised analysis on stratum corneum}

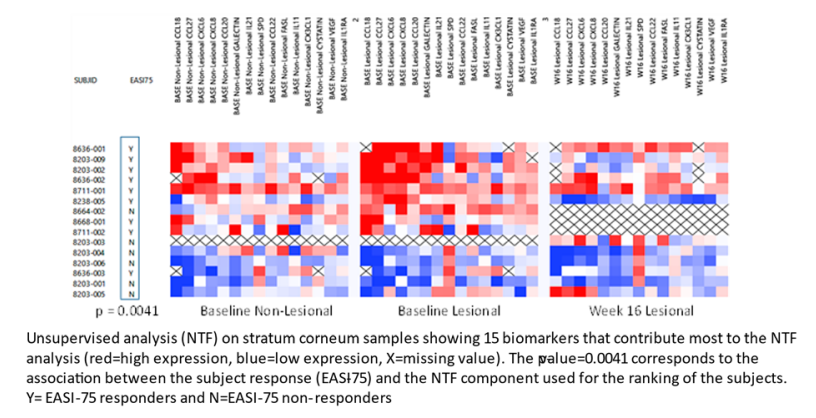

Fig. 5 Unsupervised analysis by non-negative tensor factorization (NTF) on stratum corneum samples showing fitted values from the NTF model (red = high expression, blue $=$ low expression, $\mathrm{X}=$ missing value). Proteins that

subjects (eczema, peripheral edema, and staphylococcal skin infection); those two subjects discontinued the study. There were four adverse events of special interest, which were experienced by two subjects and were infectious eczematoid dermatitis, right leg edema, staphylococcal skin infection, and bilateral leg edema. None of the special interest adverse events led to study discontinuation. show significant changes over time and skin condition appear in the left part of the heatmap, in contrast to more stable proteins that appear in the right part

\section{DISCUSSION}

This was a phase 2 , single-arm, open-label study to evaluate the PK and safety of nemolizumab in adolescents with moderate to severe $\mathrm{AD}$ and severe pruritus. The PK results demonstrated in this study are consistent with previous data from studies of nemolizumab in adults with moderate to severe $\mathrm{AD}$ and severe pruritus. 
Nemolizumab $30 \mathrm{mg}$ treatment in adolescents was associated with $C_{\max }$ at $1-2$ weeks after injection of the $60-\mathrm{mg}$ loading dose, while steady state occurred at week 4 with $C_{\text {trough }}$ ranging from $2935 \pm 1029 \mathrm{ng} / \mathrm{mL}$ to $3292 \pm 2018 \mathrm{ng} / \mathrm{mL}$ over the treatment period up to week 16. Nemolizumab observed exposures in adolescents were comparable to those in adults receiving the same dosing regimens. The PK profile of nemolizumab in adolescents was described by a one-compartment model with linear elimination and first-order absorption with a lag time. The popPK analysis confirmed a similar PK profile in adults and adolescents and predicted a mean half-life of $16.7 \pm 4.1$ days. As for adults, the main intrinsic source of PK variability on exposure in adolescents was body weight, impacting both elimination $(\mathrm{CL} / F)$ and distribution $(V / F)$, while albumin only slightly impacted $\mathrm{CL} / F$. Incidence of ADA formation in the adolescent population was limited, and no ADA-positive sample had neutralizing antibodies.

The secondary objective of the study was to evaluate the efficacy of nemolizumab and to further characterize the relationship between nemolizumab systemic exposure and clinical efficacy endpoints (PK/PD relationship). Although this study had a small sample size and was open-label, efficacy data suggest that nemolizumab improved signs/symptoms of $\mathrm{AD}$ in adolescents to a degree comparable to adults. Silverberg et al. reported a reduction in EASI score of $68.5 \%$ at week 16 in adults treated in nemolizumab, which is quite similar to the $66.5 \%$ observed in this study [17]. PK/PD models developed in adults were able to describe nemolizumab individual efficacy profiles in adolescents for clinical endpoints, providing more evidence for similarity of exposure-response in adolescents and adults. $\mathrm{AD}$ is a heterogenous disease and defining clear biomarkers of responsiveness is critical towards elucidating and comparing the mechanism of action of specific therapies. Biomarker analysis in this study clearly identified a panel of ADrelated pro-inflammatory biomarkers (including CCL17, CCL20, CCL22, CCL27, VEGF, IL-1RA, and CCL18) that are upregulated in lesional skin (as compared to non-lesional skin) and are correspondingly downregulated in clinical responders (as defined by EASI75 and PP-NRS $\geq 4$ ) of nemolizumab. These biomarkers have been previously reported as clear indicators for disease severity and are likely major players in $\mathrm{AD}$ pathogenesis. A recent review on behalf of the International Eczema Council (IEC) indicated that data for CCL17 currently has the strongest evidence for correlation with $\mathrm{AD}$ severity, but the report also discusses the roles of CCL22 and CCL27 [18]. Notably, IEC suggested that CCL22 may be the best biomarker of disease response according to studies using different treatment approaches including crisaborole, cyclosporine, and fezakinumab $[18,19]$. Interestingly, while CCL17 and CCL2 and CCL27 have been shown to attract type 2 cells to inflammatory sites $[18,19]$, CCL27 has been described to induce the migration of skin-associated memory $\mathrm{T}$ cells into cutaneous sites [18-20]. While evaluating changes in biomarkers in subjects with moderate to severe $\mathrm{AD}$ before and after oral Janus kinase/SYK inhibition, Pavel et al. observed changes in CCL20 and CCL22, among other markers [21]. Additionally, decreases in CCL17 and CCL18 by dupilumab treatment were associated with clinical improvement [19-21].

Study limitations include a small sample size, the lack of a placebo control, and the openlabel design.

\section{CONCLUSIONS}

Nemolizumab PK and safety profiles in adolescents with moderate to severe $\mathrm{AD}$ study are consistent with previous data from studies of nemolizumab in adults with moderate to severe AD. PK/PD models demonstrate similar exposure-response profiles in 12- to 17-year-old adolescents and adults for three clinical endpoints (EASI, IGA, and PP-NRS). Nemolizumab treatment reversed AD-related pro-inflammatory biomarkers in skin, indicating that the neuroimmune cytokine IL-31 is the central mediator of multiple pathways in AD. 


\section{ACKNOWLEDGEMENTS}

List of investigators. Dr Sady Alpizar, Dr William Abramovits, Dr Frank Calcagno, Dr Jamie Weisman, Dr Vivian Laquer, Dr Timothy Rodgers, Dr Sunil Dhawan, Dr William Paull, Dr Robert Call, Dr Pearl Kwong.

Funding. This study and Rapid Service Fee were funded by Galderma R\&D, Lausanne, Switzerland.

Authorship. All named authors meet the International Committee of Medical Journal Editors (ICMJE) criteria for authorship for this article, take responsibility for the integrity of the work as a whole, and have given their approval for this version to be published.

Author Contributions. All authors contributed to the study conception and design. Material preparation, data collection and analysis were performed by Dr Piketty, Mr Ahmad, Mr Loprete, Dr Krishnaswamy, and Dr JabbarLopez. The first draft of the manuscript was written by Dr Piketty and all authors commented on previous versions of the manuscript. All authors read and approved the final manuscript.

Medical Writing and/or Editorial Assistance. The authors wish to thank Valerie Sanders, from Sanders Medical Writing, for providing editorial assistance.

Disclosures. Robert Sidbury has served as an investigator for Galderma, Regeneron, Pfizer, and UCB; Sady Alpizar has served as an investigator for Galderma; Vivian Laquer has served as an investigator for Galderma and her employer received research grants; Sunil Dhawan has served as an investigator for Galderma; William Abramovits has served as an investigator for Galderma; Luca Luprete, Faiz Ahmad, Zarif Jabbar-Lopez, Jayendar Krishnaswamy and Christophe Piketty are employees of Galderma.

Compliance with Ethics Guidelines. Ethics committee approval was provided by
ADVARRA, 6940 Columbia Gateway Drive Suite\#110, Columbia, MD 21046 USA. This study was conducted in accordance with the accepted version of the Declaration of Helsinki and all relevant federal regulations as set forth in Parts 50, 56, 312, Subpart D, of Title 21 of the US Code of Federal Regulations, in compliance with International Council for Harmonization (ICH) Good Clinical Practice (GCP) guidelines, and according to the appropriate regulatory requirements in the countries where the study was conducted. Prior to entering the study, the investigator (or designee) explained to each subject (or their legally acceptable representative[s], if applicable) the nature of the study, its purpose, procedures, expected duration, alternative therapy available, and the benefits and risks involved in study participation. Subjects were given written information about the study, and, before any study procedures were performed, each subject (or their legally acceptable representative[s] if applicable) voluntarily signed and dated the informed consent form (ICF, or assent form, as applicable). All minor subjects who participated in this clinical study must have been accompanied by a parent/guardian. Subjects and guardians were required to be fully informed about the clinical study in accordance with good clinical practice guidelines, federal regulations, (for the USA, the Health Insurance Portability and Accountability Act [HIPAA, 1996]) and guidelines and in accordance with local requirements. Subjects and guardians were informed in the ICF that the results of this study may be included in publications.

Data Availability. The data that support the findings of this study are available from the corresponding author on reasonable request and with permission of the study's sponsor. The data are not publicly available due to reasons of sensitivity and other reasons.

Open Access. This article is licensed under a Creative Commons Attribution-NonCommercial 4.0 International License, which permits any non-commercial use, sharing, adaptation, distribution and reproduction in any medium or format, as long as you give appropriate credit 
to the original author(s) and the source, provide a link to the Creative Commons licence, and indicate if changes were made. The images or other third party material in this article are included in the article's Creative Commons licence, unless indicated otherwise in a credit line to the material. If material is not included in the article's Creative Commons licence and your intended use is not permitted by statutory regulation or exceeds the permitted use, you will need to obtain permission directly from the copyright holder. To view a copy of this licence, visit http://creativecommons.org/licenses/by$\mathrm{nc} / 4.0 /$.

\section{REFERENCES}

1. Weidinger S, Novak N. Atopic dermatitis. Lancet. 2016;387:1109-22.

2. Silverberg JI, Barbarot S, Gadkari A, et al. Atopic dermatitis in the pediatric population: a cross-sectional, international epidemiologic study. Ann Allergy Asthma Immunol. 2021;126:417-28 e2.

3. Eichenfield LF, Totri C. Optimizing outcomes for paediatric atopic dermatitis. $\mathrm{Br} \mathrm{J}$ Dermatol. 2014;170(Suppl 1):31-7.

4. Legat FJ. Itch in atopic dermatitis-what is new? Front Med (Lausanne). 2021;8:644760.

5. Barbarot S, Auziere S, Gadkari A, et al. Epidemiology of atopic dermatitis in adults: results from an international survey. Allergy. 2018;73:1284-93.

6. Langan SM, Irvine AD, Weidinger S. Atopic dermatitis. Lancet. 2020;396:345-60.

7. Weidinger S, Beck LA, Bieber T, Kabashima K, Irvine AD. Atopic dermatitis. Nat Rev Dis Primers. 2018;4: 1.

8. Damiani G, Calzavara-Pinton P, Stingeni L, et al. Italian guidelines for therapy of atopic dermatitisAdapted from consensus-based European guidelines for treatment of atopic eczema (atopic dermatitis). Dermatol Ther. 2019;32:e13121.

9. Sidbury R, Tom WL, Bergman JN, et al. Guidelines of care for the management of atopic dermatitis: Section 4. Prevention of disease flares and use of adjunctive therapies and approaches. J Am Acad Dermatol. 2014;71:1218-33.

10. Hansel K, Patruno C, Antonelli E, et al. Dupilumab in adolescents with moderate to severe atopic dermatitis: a 32-week real-world experience during the COVID-19 pandemic. Clin Exp Dermatol. 2021. https://doi.org/10.1111/ced.14862.

11. Stingeni L, Hansel K, Antonelli E, et al. Atopic dermatitis in adolescents: effectiveness and safety of dupilumab in a 16-week real-life experience during the COVID-19 pandemic in Italy. Dermatol Ther. 2021;34:e15035.

12. Singh B, Jegga AG, Shanmukhappa KS, et al. IL-31driven skin remodeling involves epidermal cell proliferation and thickening that lead to impaired skinbarrier function. PLoS ONE. 2016;11:e0161877.

13. Sonkoly E, Muller A, Lauerma AI, et al. IL-31: a new link between $\mathrm{T}$ cells and pruritus in atopic skin inflammation. J Allergy Clin Immunol. 2006;117:411-7.

14. Szegedi K, Kremer AE, Kezic S, et al. Increased frequencies of IL-31-producing $\mathrm{T}$ cells are found in chronic atopic dermatitis skin. Exp Dermatol. 2012;21:431-6.

15. Ruzicka T, Hanifin JM, Furue M, et al. Anti-interleukin-31 receptor A antibody for atopic dermatitis. N Engl J Med. 2017;376:826-35.

16. Silverberg JI, Pinter A, Pulka G, et al. Phase 2B randomized study of nemolizumab in adults with moderate-to-severe atopic dermatitis and severe pruritus. J Allergy Clin Immunol. 2020;145:173-82.

17. Silverberg JI, Pinter A, Alavi A, et al. Nemolizumab is associated with a rapid improvement in atopic dermatitis signs and symptoms: subpopulation $($ EASI $\geq 16)$ analysis of randomized phase $2 \mathrm{~B}$ study. J Eur Acad Dermatol Venereol. 2021. https://doi. org/10.1111/jdv.17218.

18. Renert-Yuval Y, Thyssen JP, Bissonnette R, et al. Biomarkers in atopic dermatitis-a review on behalf of the International Eczema Council. J Allergy Clin Immunol. 2021;147:1174-90 e1.

19. Glickman JW, Han J, Garcet S, Krueger JG, Pavel AB, Guttman-Yassky E. Improving evaluation of drugs in atopic dermatitis by combining clinical and molecular measures. J Allergy Clin Immunol Pract. 2020;8:3622-5 e19.

20. Tohyama M, Matsumoto A, Tsuda T, Dai X, Shiraishi K, Sayama K. Suppression of IL-17A-induced CCL20 production by cytokine inducible SH2-containing protein 1 in epidermal keratinocytes. J Dermatol Sci. 2021;101:202-9.

21. Guttman-Yassky E, Diaz A, Pavel AB, et al. Use of tape strips to detect immune and barrier abnormalities in the skin of children with early-onset atopic dermatitis. JAMA Dermatol. 2019;155: 1358-70. 